\title{
Research on Irreversible Growth Mechanism of PBX due to Thermal Cycling
}

\author{
Siyang LEI ${ }^{1, *}$, Shuo WANG ${ }^{1}$, Fangyun $\mathrm{LU}^{1}$, Rong $\mathrm{CHEN}^{1, * *}$ \\ ${ }^{1}$ College of Liberal Arts and Sciences, National University of Defense Technology, Changsha 410073, China
}

\begin{abstract}
Current experiments show that the TATB-based polymer bonded explosives (PBX) will experience irreversible growth when suffered temperature cycling load. Although some studies have already been done on this, the cause of irreversible growth is still confusing, and the mechanism is not clear. In order to study the irreversible growth of PBX under temperature cycling load, an thermal-viscoelastic model is established, Based on the Burgers model which considering the influence of different temperatures on the creep properties of PBX. The analysis shows that the irreversible growth of PBX produced by the different creep properties of high and low temperature during the thermal cycling. Comparing with low temperature, the creep rate of $\mathrm{PBX}$ is faster and the deformation is larger at high temperature, which lead to the irreversible growth of PBX.
\end{abstract}

\section{Introduction}

Polymer bonded explosive (PBX) is a heterogeneous material or mixture made of granular main explosive, high polymer binder and desensitizer by a certain process $^{[1]}$.Actually,due to diurnal variation and seasonal changes, the volume of PBX explosive increases irreversibly during thermal cycling, which is called ratchet growth $^{[2]}$.

Researchers around the world have carried out various researches on ratchet growth of PBX explosives. Mulford et $\mathrm{al}^{[3]}$ found that the sensitivity of the TATBbased explosives increased after thermal cyclings. The results of urtiew et al. ${ }^{[4]}$ showed that the increase of impact sensitivity of LX-17 explosive after thermal cycling is due to the gap produced during thermal cycling or transformed by ratchet growth. Li Wenbin et al. ${ }^{[5]}$ pointed out that the macroscopic size change and anisotropic volume expansion of PBX explosive which were caused by heated affect the overall function of warhead, and the gap between charge and shell makes it easy for the charge of weapon warhead to rub and collide with the shell in vibration, impact and vibration environment, which affects the safe use of weapon system. In addition, Tu Xiaozhen et al. ${ }^{[6]}$ put forward that studying the irreversible growth mechanism of PBX under thermal cycling is conducive to a in-depth understanding of its environmental adaptability, further improve the safety and reliability of initiating explosive devices and weapon systems, and is of great significance to analyze and predict the stability of PBX during longterm storage and delivery. Then, Willey et $\mathrm{a}^{\left[{ }^{[7]}\right.}$ found that the volume of pores in PBX increases after temperature loading, and Perry et al. ${ }^{[8]}$ proved that the voids generated during the irreversible deformation of PBX9502 are mainly intergranular cracks.

Although domestic and foreign scholars have conducted relevant research on the irreversible growth of PBX after thermal cycling and accumulated a certain theoretical basis, due to the complex microstructure, diverse process and poor service environment, the understanding of its growth mechanism in academic circles is still weak and the material constitutive model is still not complete ${ }^{[9]}$.

It is found that the ratchet growth is very similar to creep. Considering the effect of temperature on the creep properties of PBX, a theoretical model is established to analyze the irreversible deformation of PBX based on Burgers model. And the mechanism of irreversible growth is analyzed from the perspective of creep.

\section{Irreversible deformation of explosives under thermal cycling}

\subsection{A preliminary analysis of irreversible growth}

Thompson et al. ${ }^{[10,11]}$ carried out thermal cycling test on PBX9502 grains between $-43^{\circ} \mathrm{C}$ and $113^{\circ} \mathrm{C}$, and set each cycle's initial and final temperature to $23^{\circ} \mathrm{C}$. The results are shown in Figure 1,which shows that: after each cycle,the irreversible strain will be accumulated. But it tends to flatten out and no longer change in the end. In other words, it seems to have reached a certain limit.

\footnotetext{
*Corresponding author: Siyang LEI, 1403209513@qq.com, 19174166901;

**Rong CHEN, r chen@nudt.edu.cn, 15111054757
} 


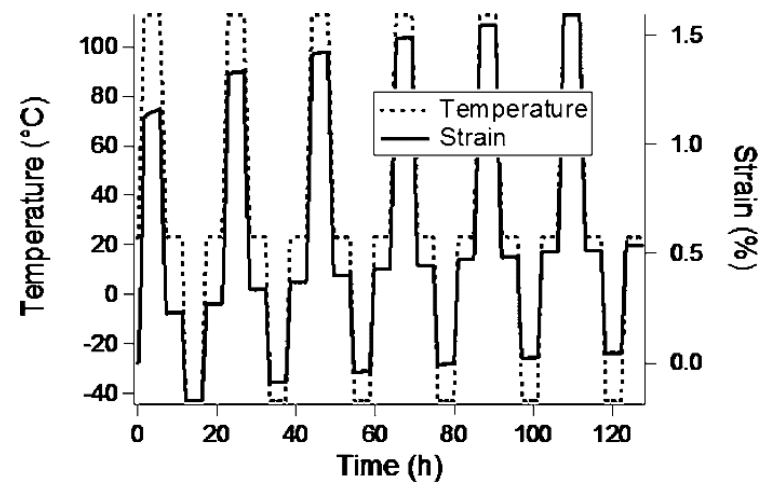

Fig. 1. Dimensional changes and irreversible growth of $P B X$ 9502 specimens under cyclic heating / cooling between $-54^{\circ} \mathrm{C}$ and $120^{\circ} \mathrm{C}^{[10,11]}$

\subsection{Analysis of internal temperature change of explosives}

The increase of temperature is caused by the heat conduction. The temperature of each position at each moment can be obtained by solving the heat conduction equation. Ignoring the influence of thermal expansion, creep and other deformation, it can be considered that the temperature change of a specific position is only a function of time, and the corresponding thermal stress and thermal expansion are the same. The ambient temperature is shown in Fig. 2.

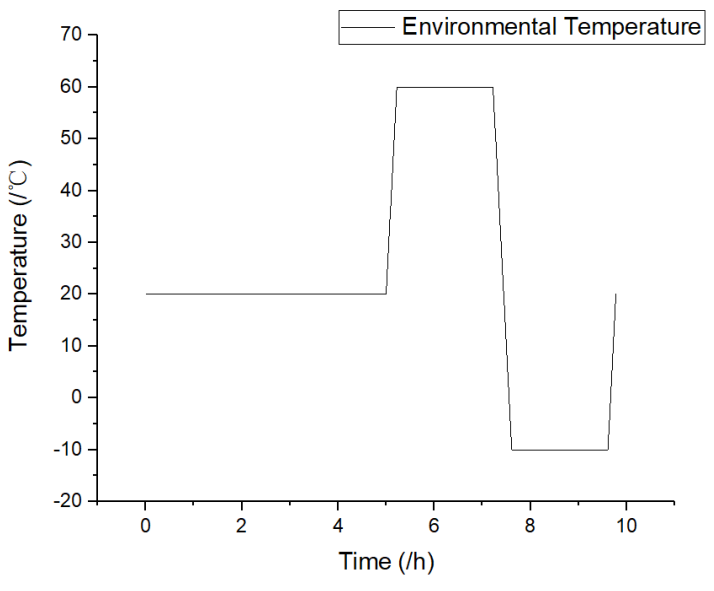

Fig. 2. Ambient temperature

Due to the low speed of temperature rise and the small size of explosive, there is little difference in the temperature of PBX grain's each position in the process of thermal cycling. Therefore, it can be considered that the internal temperature distribution in PBX is uniform.

\section{Creep strain calculation based on Burgers model}

\subsection{Calculation method of creep strain}

A Burgers viscoelastic model is shown in Figure 3:

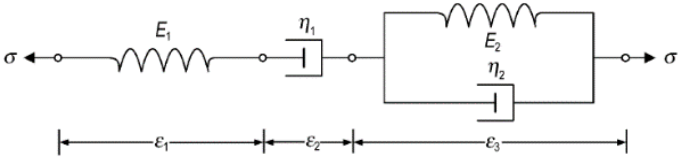

Fig. 3. Burgers viscoelastic model

The constitutive equation of Burgers viscoelastic model is

$\sigma+\left(\frac{\eta_{1}}{k_{2}}+\frac{\eta_{2}}{k_{2}}+\frac{\eta_{1}}{k_{1}}\right) \dot{\sigma}+\frac{\eta_{2}}{k_{2}} \frac{\eta_{1}}{k_{1}} \ddot{\sigma}=\eta_{1} \dot{\varepsilon}+\frac{\eta_{1} \eta_{2}}{k_{2}} \ddot{\varepsilon}$

To do laplace:

$$
P(s) \hat{\sigma}=Q(s) \hat{\varepsilon}
$$

When

$$
\left\{\begin{array}{c}
P(s)=1+p_{1} s+p_{2} s^{2} \\
Q(s)=q_{1} s+q_{2} s^{2}
\end{array}\right.
$$

Let

$$
\alpha=1-\frac{q_{1}\left(q_{2} p_{1}-q_{1} p_{2}\right)}{q_{2}^{2}}
$$

And compliance can be expressed as:

$$
\begin{aligned}
F(s) & =\frac{P(s)}{Q(s)} \\
& =\frac{p_{2}}{q_{2}}+\left(\frac{q_{2} p_{1}-q_{1} p_{2}}{q_{2}{ }^{2}}+\frac{\alpha}{q_{1}}\right) \frac{1}{s}-\frac{\alpha q_{2}}{q_{1}\left(q_{1}+q_{2} s\right)}
\end{aligned}
$$

To do inverse laplace transform:

$$
f(t)=\frac{1}{k_{1}} \delta(t)+\frac{1}{\eta_{1}}+\frac{1}{\eta_{2}} e^{-\frac{k_{2}}{\eta_{2}}}
$$

So the integral form of Burgers volume strain under variable loads is obtained:

$$
\begin{aligned}
\varepsilon(t) & =\int_{0}^{t} f(t-\xi) \sigma(\xi) d \xi \\
& =\int_{0}^{t}\left[\frac{1}{k_{1}} \delta(t)+\frac{1}{\eta_{1}}+\frac{1}{\eta_{2}} e^{-\frac{k_{2}}{\eta_{2}} t}\right] \sigma(\xi) d \xi
\end{aligned}
$$

For the loading and unloading of elasticity, the creep strain of Burgers volume $\varepsilon_{\mathrm{f}}$ can be calculated directly by the Hooke's law. And the corresponding strain response is as follow: 


$$
\varepsilon_{f}(t)=\int_{0}^{1}\left[\frac{1}{\eta_{1}}+\frac{1}{\eta_{2}} e^{-\frac{k_{2}}{\eta_{2}} t}\right] \sigma(\xi) d \xi
$$

\subsection{Parameters modification of Burgers model at different temperatures}

Parameters of Burgers model for PBX at different temperatures ${ }^{[12]}$ are given in Table 1.And the strain response of PBX under specific temperature and load is obtained, as shown in Fig. 3.

Table 1. Parameters of Burgers model for PBX

\begin{tabular}{|c|c|c|c|c|c|}
\hline$T /{ }^{\circ} \mathrm{C}$ & $\sigma_{0} / \mathrm{MPa}$ & $E_{1} / \mathrm{GPa}$ & $\eta_{1} / \mathrm{GPa}$ & $E_{2} / \mathrm{GPa}$ & $\eta_{2} / \mathrm{GPa}$ \\
\hline 24 & 5.32 & 4.8390 & $3.9394 \times 10^{7}$ & 6.4037 & $7.4479 \times 10^{5}$ \\
\hline 50 & 1.72 & 2.8538 & $2.6254 \times 10^{7}$ & 4.7164 & $4.1235 \times 10^{5}$ \\
\hline 70 & 3.45 & 2.1547 & $1.7862 \times 10^{7}$ & 1.2357 & $0.9212 \times 10^{5}$ \\
\hline
\end{tabular}

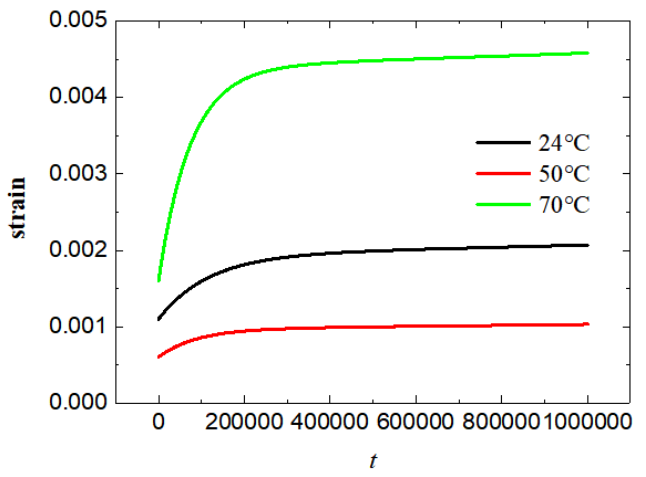

Fig. 4. Strain curves of $P B X$ under different temperature

The modulus are different at different temperatures. Although it affects the jumping point of the curves, it does not affect the subsequent creep performance. $\eta_{1}$ determines whether the material can keep rheological properties after the stress in Kelvin model is completely relaxed and its influence time is long. $\tau$ means the relaxation time. After the elastic response, stresses still be relaxed for some dozens of hours. During this time, there will be obvious creep at different temperatures and the effect on creep speed and creep strain is significantly greater than that of $\eta_{1}$. The higher the temperature is, the shorter the relaxation time is. In addition, the smaller $k_{2}$ means that the strain which can provide creep will increase significantly, while the increase of temperature leads to a significant decrease. Considering the experimental time and analysis of the above factors, we focus on the influence of temperature on $k_{2}$ and $\tau$. Then, the relationship between $T$ and $k_{2}, \tau$ are fitted linearly:

$$
\begin{gathered}
\tau=1.368 \times 10^{5}-917.5 T \\
k_{2}=(9.4-0.11 T) e^{9}
\end{gathered}
$$

\section{Calculation of strain response of explosives under variable temperature}

The creep strain of Burgers volume under variable loads is calculated by integral form:

$$
\varepsilon_{f}(t)=\int_{0}^{t}\left[\frac{1}{\eta_{1}}+\frac{1}{k_{2}[T(\xi)] * \tau[T(\xi)]} e^{\left.-\frac{t-\xi}{\tau T(\xi)]}\right]} \delta(\xi) d \xi\right.
$$

By solving the heat conduction equation, the temperature and thermal expansion strain at each position and moment are obtained:

$$
\Delta \varepsilon_{\mathrm{T}}(t)=\beta \Delta T
$$

The thermal stress in this time step is calculated as follows:

$$
\Delta \sigma(t)=\alpha k_{1} \frac{\Delta T}{T_{0}}
$$

In the formula.12 and $13, k_{1}$ is the modulus of elasticity and $\beta$ is the coefficient of thermal expansion of explosives. Assuming that they do not change with temperature, the total strain is

$$
\varepsilon(t)=\varepsilon_{\mathrm{T}}(t)+\varepsilon_{\mathrm{f}}(t)
$$

According to the creep parameters of Burgers, the strain response of explosives under alternating temperature of $-10^{\circ} \mathrm{C}$ and $60^{\circ} \mathrm{C}$ is calculated. The mode of thermal cycling is shown as Fig. 5(a). And the calculated thermal expansion strain, creep strain and total strain are shown in Fig. 5.

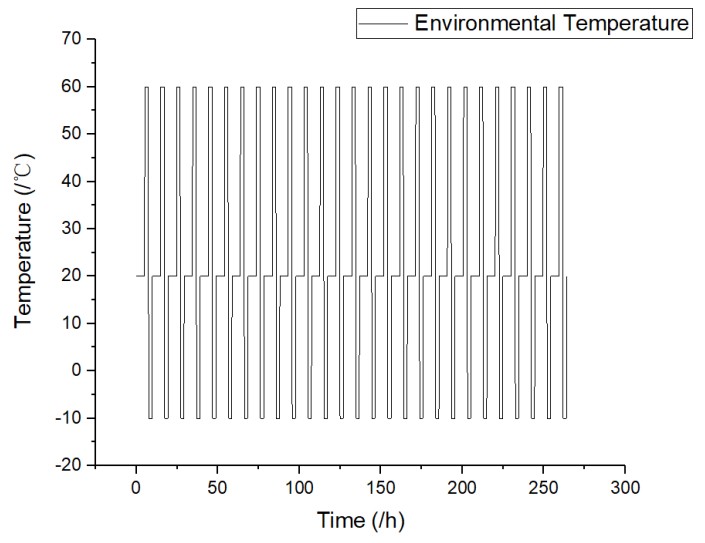

(a)The mode of thermal cycling 


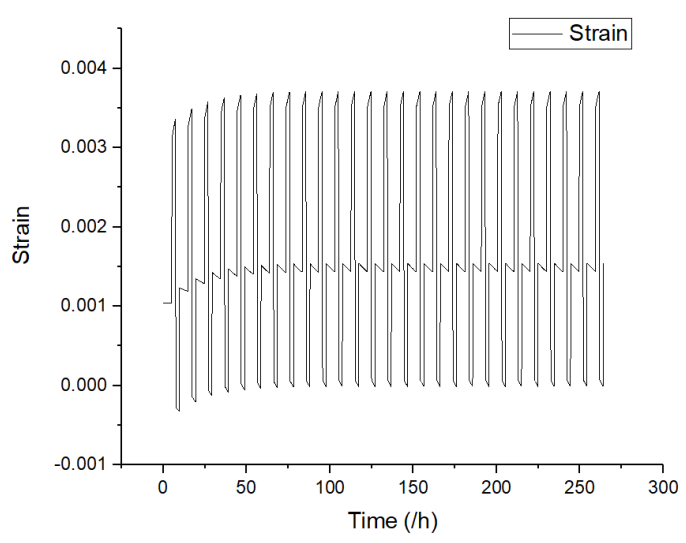

(b)Strain

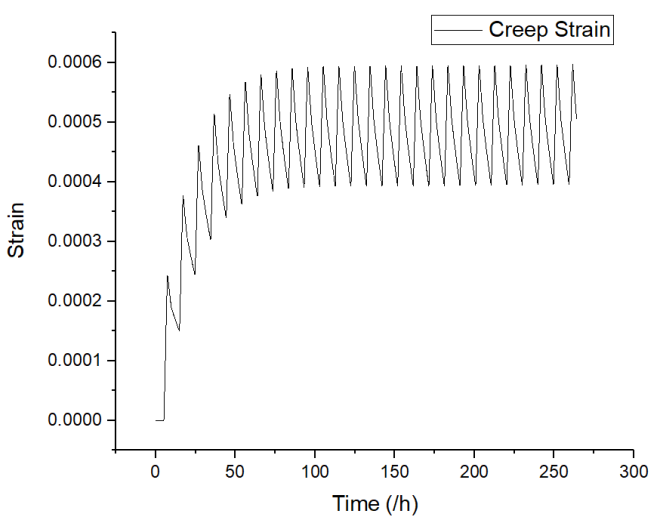

(c)Irreversible strain

Fig. 5. Strain response of $\mathrm{PBX}$ under alternating temperature $-10-60{ }^{\circ} \mathrm{C}$

According to the calculation, the strain response of explosives under temperature cycles is composed of thermal expansion and creep caused by thermal stress. And the irreversible deformation is mainly due to the different creep characteristics at high and low temperature. In Fig. 5(c): When the explosive is loaded by thermal stress at high temperature, it has large creep compliance, fast speed and large deformation. When it returns to low temperature, the thermal stress is unloaded and the explosive flexibility is small. In addition, the creep speed is slow and the deformation is small, which leads to irreversible creep deformation in a single cycle. Combined with Fig. 4, the slope of each creep curve in the middle and later stages is obviously decreases and gradually converges. It is considered that the reason is the gradual hardening of explosives. At that time, the creep compliance decreases with the continuous loading and creep deformation. After several temperature cycles, due to the hardening effect, the creep compliance of explosives at high and low temperatures will gradually approach.

\section{Conclusions}

Based on Burgers model which describes the creep behavior of viscoelastic materials, considering the influence of different temperatures on the creep characteristics of PBX explosive, an analytical model for the deformation and irreversible growth of explosive in temperature cycle is established. And the strain response and irreversible deformation of PBX under temperature cycling are calculated by this model, which is consistent with the observed phenomena in references ${ }^{[11,12]}$.And this paper further analyzes the main causes of irreversible deformation of PBX: Due to the difference of creep characteristics between high and low temperature stages under thermal cycling, PBX has fast creep and large deformation at high temperature stage, while slow creep and small deformation at low temperature stage. It leads to irreversible creep deformation in the process of thermal cycling.

\section{Acknowledgments}

The National Natural Science Fund (No.12072369)

\section{References}

1. S. Ye,K. Tonokura,M. Koshi.Energy transfer rates and impact sensitivities of crystalline explosives[J]. Combust. Flame(2003),132(1-2):240-246.

2. Y.B. LI,F.D. NIE,J. SUN, et al,Influence of Irreversible Growth of PBX on Interface of TATB/binder[J].Chin.J.Explos.Propellants(2001).24 (4).

3. R.N. Mulford and D.C. Swift.Sensitivity of PBX9502 after ratchet growth[C].AIP Conf. Proc(2012).

4. J Neubauer,R Sieber,H Kuzel,M Ecker,W.C. Tao.Effect of confinement and thermal cycling on the shock initiation of LX-17[J],Combust. Flame (1996), 105,43.

5. W LI,X WANG,G ZHAO,et al.The research of the effect of base gap on the stress of explosives and the lunching safety[J].J. Ballist.(2001),13(3).

6. X TU,B ZHANG,W WANG,et al.Study on the Properties of TATB Based PBX Under Mechanical Stress Undergoing High Temperature Accelerated Aging Test[C].6th Symposium on Energetic Materials and Insensitive Munitions(2014).

7. T.M. Willey,T.V. Buuren,J.R.I. Lee,et al.Changes in pore size distribution upon thermal cycling of TATB-based explosives measured by ultra-small angle X-ray scattering[J]. Propellants, Explos., Pyrotech(2010), 31(6):466-471.

8. W.L. Perry, A.M. Duque, J.T. Mang, E. Roemer, New insights to the irreversible thermal expansion of PBX 9502[C],15th International Detonation Symposium, San Francisco,CA,July(2014).

9. R.B. Schwarz, Cheng Liu and Darla G.Thompson. Anisotropy in the Ratchet Growth of PBX 9502[R]. LA-UR-15-21827

10. D.G Thompson,R.B Schwarz,G.W Brown,et al. Time-Evolution of TATB-Based Irreversible Thermal Expansion (Ratchet Growth)[J]. Propellants, Explos., Pyrotech(2015), 40(4):558-565.

11. D.G. Thompson,R. DeLuca,C.S. Woznick.FY19 Summary Report: Thermal and Mechanical 
Characterization of DAAF and LAX-133, LA-UR19-29255.

12. P LI, Z.M HAO, Y. P LIU, et al. Application of Peridynamic Method on Prediction of Creep Behavior of Polymer Bonded Explosives[J]. Chin J Energ Mater(2017), 25(02):118-124. 